\title{
Jejunoileal Atresia Type IIIC: A New Variant
}

\author{
Pooja Tiwari*
}

Ms, Mch Pediatric Surgery, DNB Pediatric Surgery, MNAMS, Consultant Pediatric Surgeon, Miracles Child Care, 20 B Sainath Colony, Indore, MP, India

DOI: $10.36347 /$ sjmcr.2021.v09i01.013

| Received: 22.12.2020 | Accepted: 07.01.2021 | Published: 15.01.2021

*Corresponding author: Pooja Tiwari

Abstract

Jejunoileal atresia (JIA) and stenosis are the most common congenital anomalies of the small intestine and are a major cause of intestinal obstruction in neonates. The traditional classification of JIA proposed by Grosfeld included 4 subtypes of which type IIIB, or apple-peel atresia, is characterized by a proximal atretic jejunum and a distal segment of spiralled bowel that terminates at the cecum. These patients are at increased risk for short bowel syndrome and subsequent intestinal failure. We hereby describe a case of a neonate with JIA who was initially found to have a type IIIB atresia but there was a variation as she had a length of normal small bowel and mesentery proximal to cecum attached distal to spiralled segment of bowel. Surgical correction proceeded with minimal resection and primary anastomosis. She recovered well from this procedure, tolerated full enteral nutrition by mouth, and displayed good weight gain at outpatient follow-up. Due to the unique anatomy of the gastrointestinal tract in this case report, we propose the addition of a new class of JIA, type IIIC, to better reflect its prognostication and surgical management.

Keywords: Jejunoileal atresia; apple-peel atresia; short bowel syndrome.

Copyright $(\mathcal{C} 2021$ The Author(s): This is an open-access article distributed under the terms of the Creative Commons Attribution 4.0 International License (CC BY-NC 4.0) which permits unrestricted use, distribution, and reproduction in any medium for non-commercial use provided the original author and source are credited.

\section{INTRODUCTION}

Jejunoileal atresia (JIA) is the most common congenital anomaly of the small intestine and is a major cause of intestinal obstruction in neonates with an incidence of $1-3$ in 10,000 live births [1]. Studies suggest that JIA results from intrauterine vascular accidents [2]. Jejunoileal atresias are nearly equally distributed between the jejunum (51\%) and the ileum $(49 \%)$. Most atresias (36\%) occur in the distal ileum; $13 \%$ occur in the proximal ileum; $31 \%$ occur in the proximal jejunum; and $20 \%$ occur in the distal jejunum. Bland-Sutton and Spriggs first classified JIA in the late nineteenth and early twentieth century [3, 4]. In 1955 Louw and Barnard described three different types of jejunoileal atresia [5]. Martin and Zerella introduced the variant of multiple and apple-peel atresia [6], which were combined with the Louw categories by Grosfeld in 1979 [7] and have since remained the standard classification system for JIA. Type I atresia is characterized by a transluminal septum; type II involves a fibrous cord connecting 2 blind-ending pouches; type IIIa has a V-shaped mesenteric defect; and type IV exhibits multiple atretic segments. A special class of intestinal atresia, type IIIB, which occurs in less than $10 \%$ of all JIA [8], consists of proximal jejunal atresia and a short segment of ileum spiraling around a single mesenteric vessel. This is a case report of a variant of type IIIB JIA where a significant amount of normal ileum continued from the "apple peel/ Christmas tree" segment up to the ileocecal valve. To our knowledge, only one such case have been reported in the literature [9].

\section{Case Report}

The full-term $2.7 \mathrm{~kg}$ female neonate presented on day 3 of life with complaints of intolerance to feeds and bilious vomiting. The child was born with normal vaginal delivery with no antenatal ultrasound done. The parents where from low socioeconomic status. Physical examination on presentation revealed a soft moderately distended abdomen with visible bowel loops. She was kept nil by mouth (NBM) and a nasogastric tube (NGT) was placed to decompress stomach which had bilious aspirates. Abdominal radiograph was suggestive of jejunal atresia (Figure-1). The neonate had early onset sepsis and had low platelet counts.

After stabilisation, she was taken to the operating room for management of her presumed jejunal atresia. On exploration, the a classic distal jejunal atresia was encountered around 25 centimetres distal to the ligament of Treitz accompanied by a dilated jejunal bulb (Figure-2). The distal bowel was spiralled around a single vessel for around 50 centimetres consistent with a type IIIB or apple-peel atresia (Figure-3). But in addition to spiralled bowel 
Pooja Tiwari., Sch J Med Case Rep, Jan, 2021; 9(1): 48-50

there was around 75 centimetres of normal ileum terminating into ileocecal valve (Figure-4). The remainder of the small bowel and colon was anatomically normal and there was no malrotation. The dilated and atretic proximal bowel was resected. Distal bowel patency was confirmed and distal atresia was ruled out by flushing with saline and inspecting its free flow into distal loops of bowel. Bowel continuity was established with an end-to-end primary anastomosis by Benson technique (Figure-5) Pathological examination of the resected small bowel segment showed no obstruction or any other abnormality.

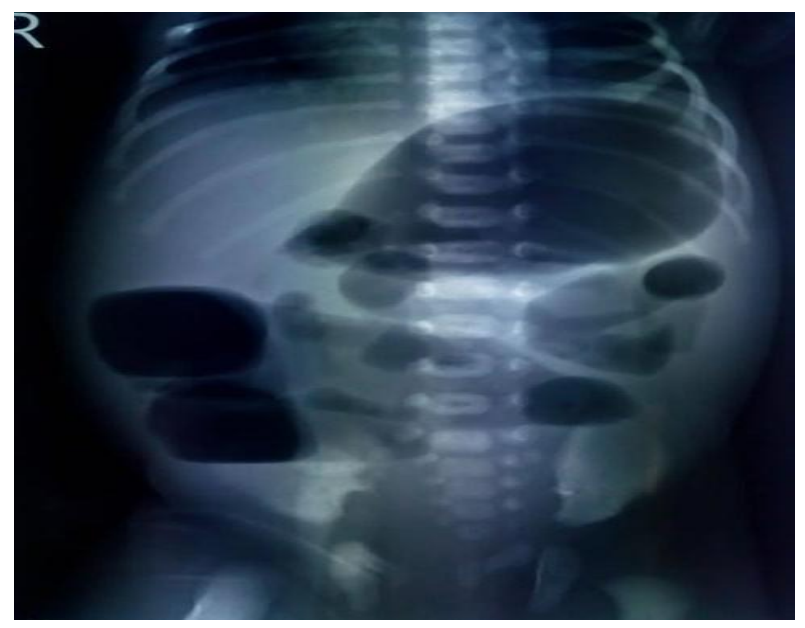

Fig-1

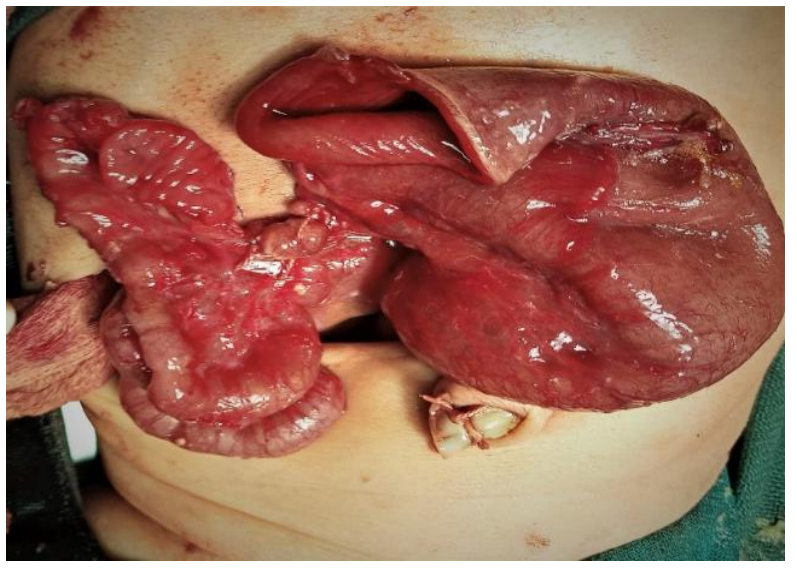

Fig-2

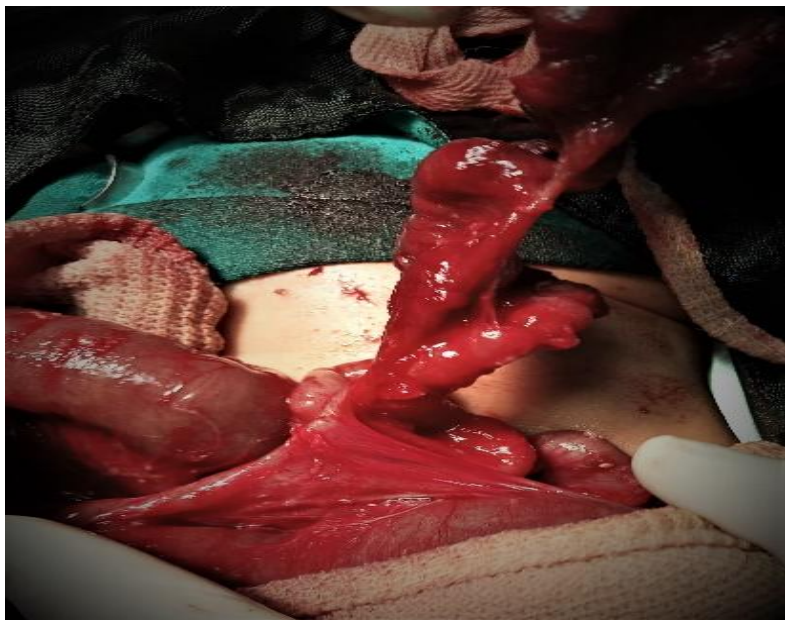

Fig-3

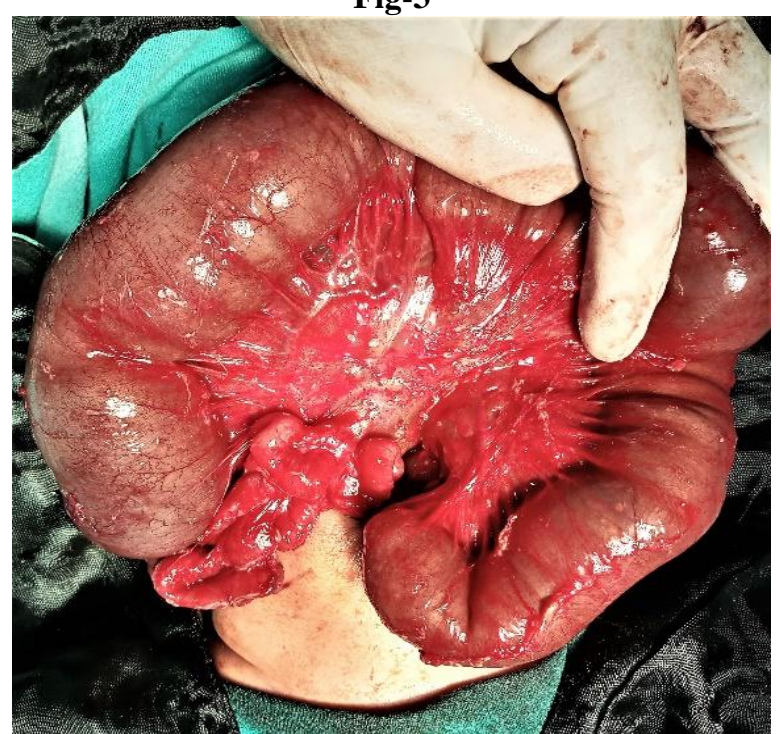

Fig-4

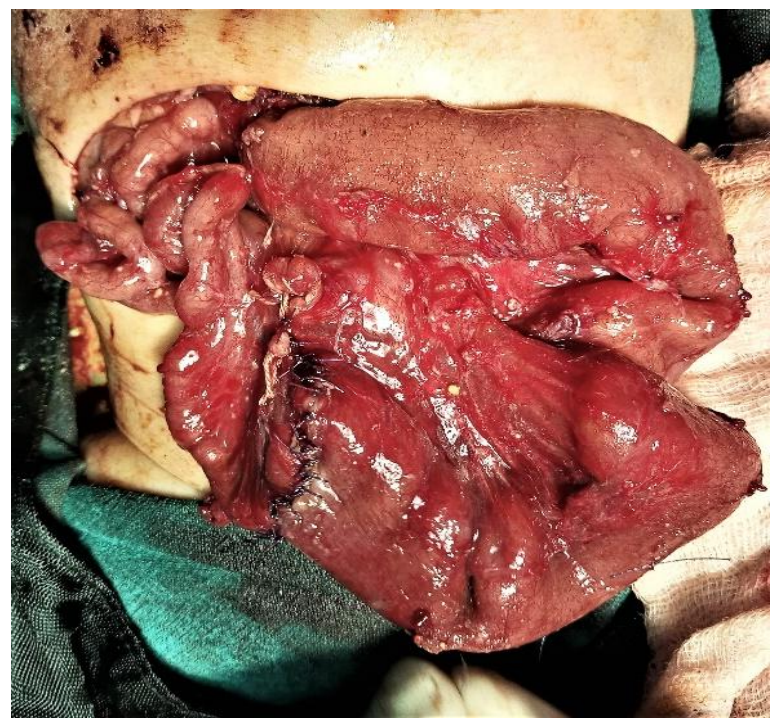

Fig-5

The patient required prolonged NBM after surgery and was kept on parenteral nutrition with monitoring of blood sugar, serum electrolytes and liver function test. Her bowel movements started on 
postoperative day (POD) 7 and test feeds breast milk was subsequently initiated via a nasal feeding tube. Her feeds were gradually advanced, and she achieved full tube enteral feed on POD 15. She was transitioned to full oral intake on POD 24 and subsequently discharged on POD 32.

On follow up, child accepted feeds well and had adequate weight gain. She attained her milestones on time in her follow up of 1 year.

\section{DISCUSSION}

Though operative mortality for operated cases of JIA has reduced over years still its association with short bowel syndrome (SBS) continue to pose clinical challenges [8, 10]. Type IIIB and IV are more commonly associated with SBS, which can occur in up to $1 / 3$ of these cases $[8,11]$. The lack of bowel length in apple peel atresia necessitates creative bowelpreserving procedures to minimize the risk of developing SBS [12]. In such unusual cases of applepeel atresia as index case, there is adequate length of bowel distal to apple peel which avoids the need for bowel preservation strategies. As there was around 75 centimetres of healthy bowel distal to apple peel, we resected the apple peel portion of bowel and anastomosed proximal healthy bowel to distal one. Even in the face of SBS, long-term outcome in patients with type IIIB atresia proves satisfactory [13]. So in the cases as index case we expect excellent prognosis as they have near normal bowel components.

Dao et al presented this unique variant of JIA first time in 2019 where the gastrointestinal tract anatomy did not fit with any of the Grosfeld classes and proposed the addition of a new class - type IIIC - to describe this variant of JIA [9]. We report a similar case of JIA and recommend the addition of new class type III $\mathrm{C}$ which can enhance our understanding of the disease, and improve its management and prognostic values.

\section{REFERENCES}

1. Best KE, Tennant PW, Addor MC, Bianchi F, Boyd P, Calzolari E, Dias CM, Doray B, Draper
E, Garne E, Gatt M. Epidemiology of small intestinal atresia in Europe: a register-based study. Archives of Disease in Childhood-Fetal and Neonatal Edition. 2012 Sep 1;97(5):F353-8.

2. Louw JH, Barnard CN. Congenital intestinal atresia; observations on its origin. Lancet (London, England). 1955; 269:1065-7.

3. Sutton JB. Imperforate ileum. Am J M Sc. 1889; 98.

4. Spriggs NI. Congenital intestinal occlusions. Guy's Hosp Rep. 1912; 66.

5. Louw JH. Resection and end-to-end anastomosis in the management of atresia and stenosis of the small bowel. Surgery. 1967; 62:940-50.

6. Martin LW, Zerella JT. Jejunoileal atresia: a proposed classification. J Pediatr Surg. 1976; 11:399-403.

7. Grosfeld JL, Ballantine TV, Shoemaker R. Operative management of intestinal atresia and stenosis based on pathologic findings. J Pediatr Surg. 1979; 14:368-75.

8. Stollman TH, de Blaauw I, Wijnen $\mathrm{MH}$, van der Staak FH, Rieu PN, Jos MT, Wijnen RM. Decreased mortality but increased morbidity in neonates with jejunoileal atresia; a study of 114 cases over a 34-year period. Journal of pediatric surgery. 2009 Jan 1;44(1):217-21.

9. Dao DT, Demehri FR, Barnewolt CE, Buchmiller TL. A new variant of type III jejunoileal atresia. Journal of pediatric surgery. 2019 Jun 1;54(6):1257-60.

10. Dalla Vecchia LK, Grosfeld JL, West KW, Rescorla FJ, Scherer LR, Engum SA. Intestinal Atresia and Stenosis. Arch Surg. 1998; 133:490-7.

11. Lee SH, Cho YH, Kim HY, Park JH, Byun SY. Clinical experience of complex jejunal atresia. Pediatr Surg Int. 2012; 28:1079-83. doi:10.1007/s00383-012-3131-5.

12. Onofre LS, Maranhão RF de A, Martins ECS, Fachin CG, Martins JL. Apple-peel intestinal atresia: Enteroplasty for intestinal lengthening and primary anastomosis. J Pediatr Surg. 2013; 48:e57.

13. Waldhausen JH, Sawin RS. Improved long-term outcome for patients with jejunoileal apple peel atresia. J Pediatr Surg. 1997; 32:1307-9. 